\title{
VALIDATION OF INNOVATIVE SYSTEMS OF REMOTE GAS LEAKS DETECTION AND QUANTIFICATION REDUCING EMISSIONS AND INCREASING SAFETY
}

Xavier Watremez, Thierry Baron, André Marblé, Véronique Miegebielle, Hélène Bideaud, ${ }^{(1)}$, Xavier Marcarian $^{(2)}$, Pierre-Yves Foucher ${ }^{(3)}$, Nicolas Cézard and Myriam Raybaut ${ }^{(4)}$

(1) TOTAL, CST-JF, avenue Larribau, 64018 Pau Cedex, France, email : xavier.watremez@total.com

(2) TOTAL, Pôle d'Etudes et de Recherche de Lacq (PERL), BP 47, 64170 Lacq, France

(3) ONERA, 2 avenue Edouard Belin, 31400 Toulouse, France

(4) ONERA, Chemin de la Hunière, BP 80100, 91123 Palaiseau Cedex France

\begin{abstract}
Gas leaks are a major issue for Oil \& Gas companies, either for environment or safety purposes. Remote sensing technologies can be applied for a wide range of gas leak flowrates and in three main cases: major leaks in crisis management; medium size leaks in safety monitoring; small leaks in environmental monitoring.

In 2019, TOTAL completed a five-year Research \& Development collaborative project NAOMI with ONERA, The French Aerospace Lab, to develop technologies for remote detection, identification, visualization and quantification of gas leaks with applications relating to safety and environmental protection.

TOTAL organized several gas test campaigns on Lacq Pilot Platform in France, called TADI (Total Anomaly Detection Initiatives) to evaluate and select relevant technologies.
\end{abstract}

Index Terms : remote sensing, gas leaks, detection, localization, quantification, hyperspectral, multispectral infrared camera, Light Detection And Ranging (Lidar), optical, acoustic

\section{INTRODUCTION}

In NAOMI project, TOTAL and ONERA have developed prototypes and software for fixed and mobile solutions of methane emission measurement technologies from environmental monitoring of fugitive emissions to monitoring and management of accidental gas leaks.

TOTAL has built its TADI (Total Anomaly Detection Initiatives) testing platform in Lacq to assess all the innovative tools in the remote gas leaks detection and quantification.

Several test campaigns were performed since 2015 [1] [2] [3]. The aim is to catalyze developments in open innovation mode by having all the players on the same controlled tests (research and development, service providers, industrialists).

\section{TADI}

TADI, located in Lacq, France, is an open-air test site measuring 2,000 $\mathrm{m}^{2}$ for qualifying systems designed for gas leaks remote observation including airplane, drones, robots and artificial intelligence processing. Equipped with surface facilities (pipelines, columns, recovered wellheads, etc.), this test area can reproduce around 30 scenarios of controlled emissions from 0.1 to $300 \mathrm{~g} / \mathrm{s}$ of gas (methane, carbon dioxide, nitrogen, and other interferent gasses such as ethane...) in an industrial environment. TADI is an important bridge between laboratory development and industrial pilots by providing the opportunity to evaluate gas leaks detection systems in a repeatable series of tests at mock facilities where the emission locations and flowrates can be controlled and metered. The TADI infrastructure is open to anyone who wants to test technologies in gas detection, quantification and localization.

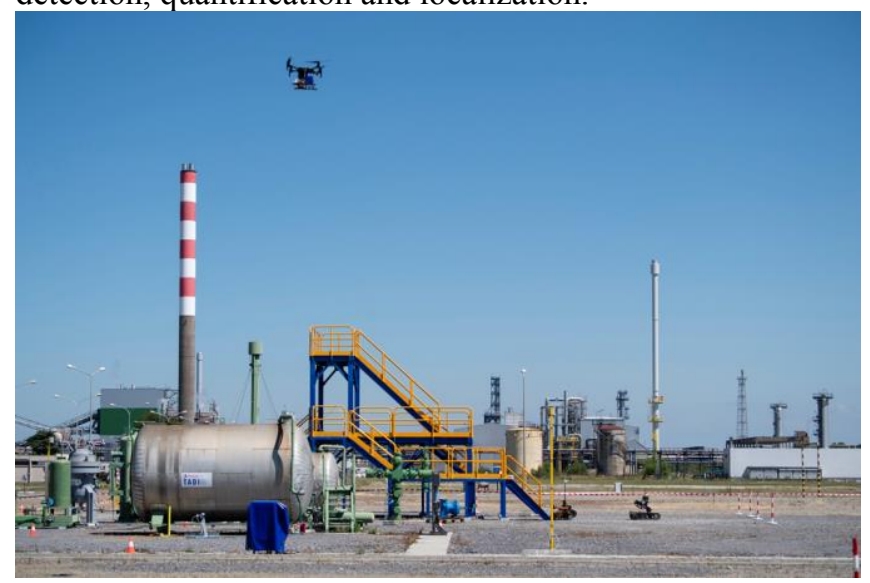

Fig. 1: Picture of TADI in Lacq, France

In 2018, a collaboration between the Methane Emission Technology Evaluation Center (METEC) at Colorado State University and TOTAL has permitted to contribute to the preparation of the tests facilities in TADI. 
The TADI test campaign in October 2018 permitted to test successfully the NAOMI prototypes : (1) multispectral infrared camera : SIMAGAZ ; (2) hyperspectral tools for real time quantification of gas flowrates (ground and airborne) : IMGSPEC ; (3) multi-gas (methane and dioxide of carbon) Lidar (LIght Detection And Ranging) system coupled with a wind Lidar : GAZ-L ; (4) combined methane and wind function Lidar: VEGA ; (5) several other remote systems from different worldwide teams were present to assess the performances of their gas quantification systems ( 25 companies present).

The latest TADI test campaign, in October 2019, validated functional solutions of remote detection methodologies for the scenario of the safety monitoring, including successful tests of the SIMAGAZ camera prototype on a drone, in handheld and fixed solution.

Networks of acoustic systems were also developed and tested on TADI to have complementary solutions of early remote detection and localization of gas leaks.

\section{MULTISPECTRAL INFRARED CAMERA FOR METHANE : SIMAGAZ}

The infrared cameras currently available on the market are capable of detecting the presence of gas, but do not provide quantitative data. Multispectral imaging is a proven technique for gas leak quantification. Measuring signal in several spectral bands (rather than in a single band) is a way to reduce the rate of false alarms. The multispectral solution, based on four bands (instead of one hundred as in hyperspectral technology), resolves the problems of weight and volume for airborne applications.

The SIMAGAZ camera was developed by ONERA in collaboration with TOTAL as part of the NAOMI project. A cooled infrared detector of the camera is provided by LYNRED, the electronics is realized by NOXANT. This camera is the first multispectral infrared mobile system which can remotely detect, localize, visualize and quantify methane leaks in real time.

Having a detection with a very high variability of gas flowrates (beginning from $1 \mathrm{~g} / \mathrm{s}$ of methane at $100 \mathrm{~m}$.), the SIMAGAZ camera can handle the three main cases : from the major leaks, and the medium size leaks increasing safety to the small leaks reducing methane emissions.

With a weight below $1.5 \mathrm{~kg}$, the SIMAGAZ was tested in the TADI test campaigns in fixed and mobile applications : handheld on ground and on board of a drone.

Presently, the camera is in the pre-industrial prototype stage for the UAV applications in two scenarios: fixed-point checks and mapping-location-quantification of gas emission sources.

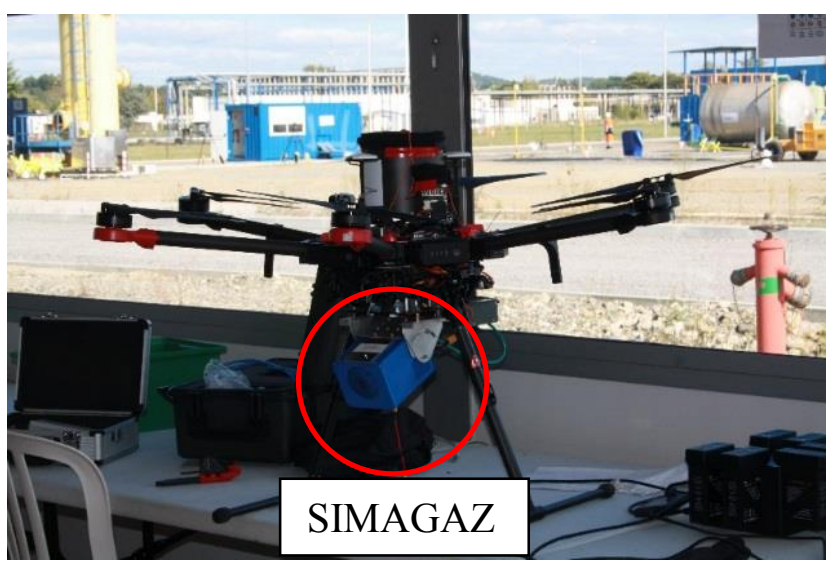

Fig. 2: Picture of SIMAGAZ multispectral camera on a drone in the TADI test campaign in October 2019

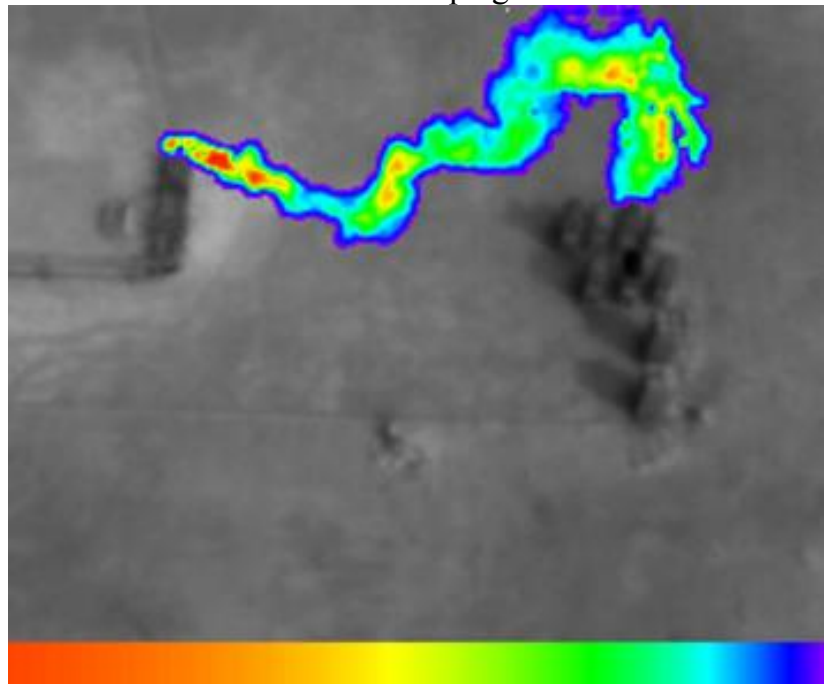

Fig. 3: Examples of maps in integrated concentrations of methane (ppm.m) by SIMAGAZ on drone in the TADI test campaign in October 2019. Non-linear scale between 0 and 20000 ppm.m of methane.

\section{REAL TIME QUANTIFICATION ALGORITHM : IMGSPEC}

A hyperspectral processing software, called "IMGSPEC", was developed, as part of the NAOMI project, for quantifying methane plumes in real time, using a hyperspectral camera. The quantification results obtained are very reliable thanks to the large number of spectral bands of the camera. The airborne application of this system is a good solution to quantify remotely major gas leaks during an emergency situation.

The algorithm IMGSPEC was validated in the TADI test campaign in June 2017 with the hyperspectral cameras of TELOPS, HyperCam, on ground. The quantification results of linear concentrations of methane produced in real time were highly satisfactory [4]. 
In the TADI test campaign in October 2018, the IMGSPEC algorithm was developed and transferred to the TELOPS HyperCam camera for airborne and ground acquisitions. Two half-days were allocated to airborne data acquisition. 69 passes were performed over the different leakages. The gas plume was detected in two thirds of passes. The HyperCam camera was installed onboard a plane. TELOPS had an airborne system adapted to its HyperCam model that comprises a gyro-stabilized platform. Flights were planned at different altitudes: between 450 and $1,250 \mathrm{~m}$ above the ground, to measure the performance range of detection and quantification operations.

The various tests performed during the campaign demonstrated the ability of a TELOPS hyperspectral camera coupled with the IMGSPEC algorithm, to visualize and quantify, remotely and in real-time, leaks of methane.

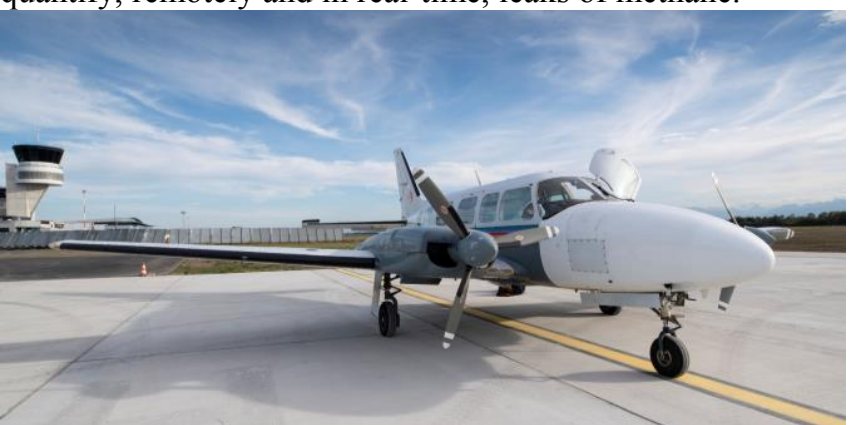

Fig. 4: Aircraft used in the TADI campaign in October 2018

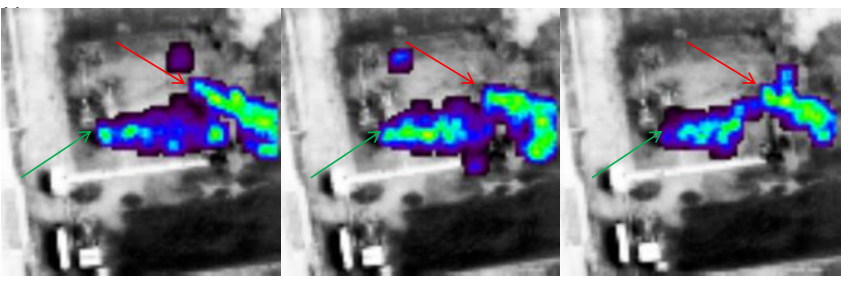

Fig. 5: Methane plume quantitative images from airborne hyperspectral data at an altitude of $600 \mathrm{~m}$ with a multi-leaks of $45 \mathrm{~g} / \mathrm{s}$ (red arrows) and $8.5 \mathrm{~g} / \mathrm{s}$ (green arrows) in the TADI campaign in October 2018

\section{GAZ-L LIDAR AND VEGA LIDAR}

In addition to passive optical systems, such as the infrared imagers (SIMAGAZ camera and IMGSPEC algorithm), active optical systems, such as Lidars (LIght Detection And Ranging) were developed in NAOMI project for remote gas detection and quantification : the GAZ-L lidar and the VEGA lidar. The compactness of these lidar, with a volume of less than $2 \mathrm{~m}^{3}$, to ease logistics of transport, is part of the innovation. The demonstrators of these lidars have been successfully tested in the TADI test campaign in October 2018 [5] [6].

The GAZ-L lidar is made of a coupling of one multi-gas (methane and dioxide of carbon) DIAL lidar and one separate Wind Doppler lidar for the measurement of the gas flows. The assembly of the prototype is composed of an emission module including the laser source, a reception module including the detector and an acquisition and treatment module. In a DIAL (DIfferential Absorption Lidar), at least two laser pulses are emitted, at two wavelengths called ON (in coincidence with one of the targeted species absorption line) and OFF (on an absorption minimum). The laser pulses are backscattered by the atmosphere, collected and detected by the DIAL receiver, and a gas plume can be localized and quantified along the line of sight by comparing the backscattered ON and OFF signals.

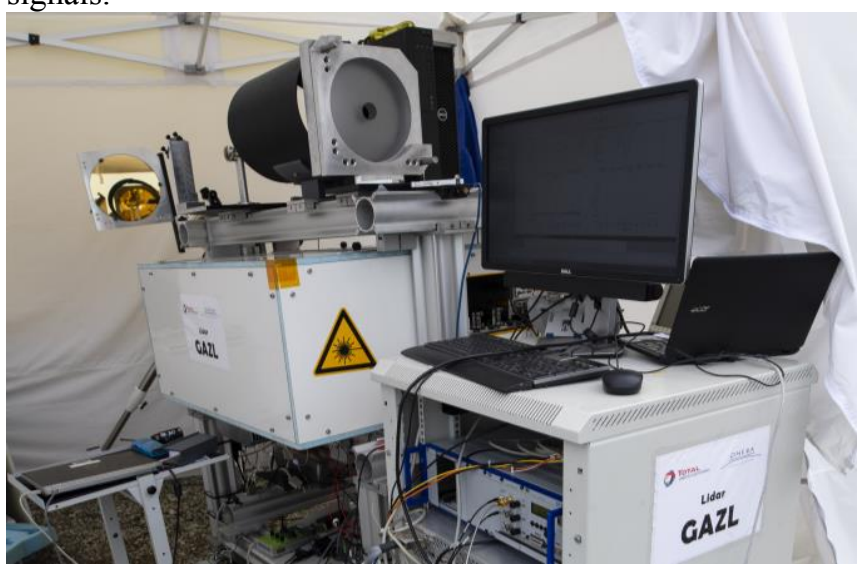

Fig. 6: Picture of GAZ-L multi-gas DIAL Lidar in the TADI campaign in October 2018
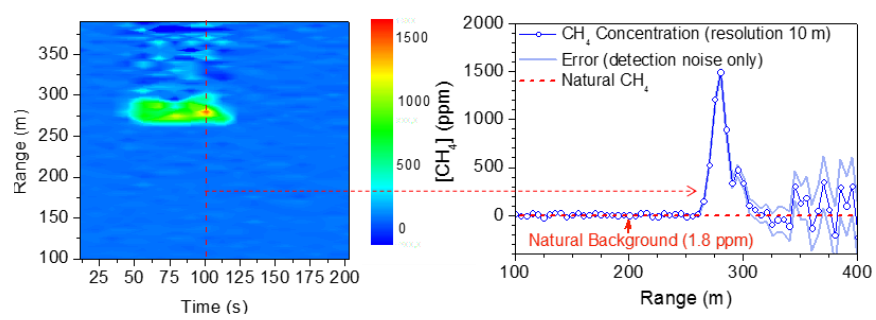

Fig. 7: Example of measurement performed in the TADI test campaign in October 2018 using GAZ-L DIAL: a controlled release of $200 \mathrm{~g} / \mathrm{s}$ of methane was generated 270

$m$ away from the DIAL. The estimated methane plume concentration on a line of sight with a $10 \mathrm{~m}$ range resolution and a $10 \mathrm{~s}$ temporal resolution is shown on the left curve

The VEGA lidar, specific to the measurement of methane flow, based on compact and robust technology bricks (fiber lasers), is composed of a single lidar with two simultaneous functions, methane DIAL and Wind Doppler, based on coherent detection. During the test campaigns in TADI in October 2018 and 2019, the VEGA lidar proved to be robust and reliable on the field, showing no significant performance variations during both campaigns, despite important temperature and humidity variations. Its implementation in TADI took around 2 hours for the first day of each campaign and around $15 \mathrm{~min}$ the following days. The expected range of distance of detection of methane for the design of this lidar is $800 \mathrm{~m}$. 


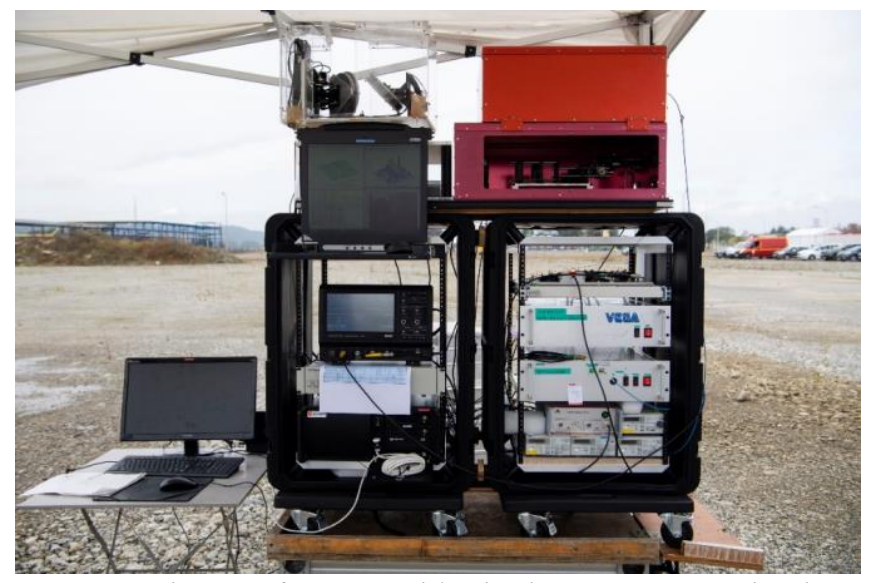

Fig. 8: Picture of VEGA Lidar in the TADI campaign in October 2018

\section{ACOUSTIC SYSTEMS}

Two senses can be used to optimize gas detection : sight and hearing. The R\&D of TOTAL is testing optical and acoustic technologies in parallel. Further to an acoustic campaign in TADI in June 2018 with five companies, two acoustic solutions have been selected by TOTAL to be developed and tested in longer period in TADI and in SOBEGI in Lacq, France since June 2019 : SIM ENGINEERING / WAVELY and ACOEM / METRAVIB. Both systems are a network of stationary microphones combining with an algorithm of machine learning to detect, locate and classify gas emissions. These systems identify the acoustic signatures of different noise sources to determine a probability of gas leak. The next step is the deployment of these acoustic systems in industrial pilots in oil and gas facilities in 2020 .

\section{CONCLUSION}

Optical and acoustic innovative systems open new perspectives for industrial and ambient monitoring covering three main cases: major leaks in crisis management; medium size leaks in safety monitoring; small leaks in environmental monitoring. In the second case of the safety monitoring, optical and acoustic innovative systems could complement, as a first step, and replace, as a second step, the existing gas detection systems of a plant to reduce maintenance activities of the existing gas detectors.

\section{ACKNOWLEDGMENTS}

The authors are very grateful to everyone involved in the NAOMI project, in particular the ONERA (French Aerospace Lab) and TOTAL teams. Part of the work on SIMAGAZ has been funded by FUI IMAGAZ and its partners. Authors are very grateful to all those who participated in the tests campaigns in TADI since 2015, in particular the TOTAL teams at the PERL (Platform for
Experimental Research in Lacq), SOBEGI, ASLINDUSLACQ, CITBA, INFAUTELEC, ONERA, TELOPS, ADCIS, MODIS, DIMAP, PIXAIR SURVEY, BERTIN TECHNOLOGIES, SENSIA, IMC SERVICE, FLIR, REBELLION PHOTONICS, LYNRED, NOXANT, HARRIS, CI SYSTEMS, PERGAM SUISSE, GAS OPTICS, DRONEVOLT, SIM ENGINEERING, WAVELY, ACOEM, PHONOPTICS, XARION, FLUKE, DISTRAN, TAUROB, CNRS, GSMA, URCA, LSCE, IFPEN, PPID, FLYLOGIX, APIX.

\section{REFERENCES}

[1] X. Watremez, N. Labat, G. Audouin, B. Lejay, X. Marcarian, D. Dubucq, A. Marblé, TOTAL; , P.Y. Foucher, L. Poutier, ONERA; R. Danno, D. Elie, ADCIS; M. Chamberland, Telops, "Remote Detection and Flow rates Quantification of Methane Releases Using Infrared Camera Technology And 3D Reconstruction Algorithm", In Proceedings of the Society of Petroleum Engineers, SPE Annual Technical Conference and Exhibition, Dubai, UAE, 26-28 September 2016

[2] X. Watremez, N. . Labat, G. Audouin, B. Lejay, X. Marcarian, D. Dubucq, A. Marblé, TOTAL; P.Y. Foucher, L. Poutier, ONERA; R. Danno, D. Elie, ADCIS, "Remote Sensing Technologies For Gas Leak Detection, Visualisation And Quantification Using Infrared Imagers", In Proceedings of the Society of Petroleum Engineers, Abu Dhabi International Petroleum Exhibition and Conference, Abu Dhabi, UAE, 7-10 November 2016

[3] X. Watremez, A. Marblé, T. Baron, X. Marcarian, D. Dubucq, L. Donnat, L.Cazes, TOTAL; P.Y. Foucher, ONERA; R. Danno, D. Elie, ADCIS; M. Chamberland, J.P. Gagnon, Telops; et al. "Remote Sensing Technologies For Detecting, Visualizing and Quantifying Gas Leaks", In Proceedings of the Society of Petroleum Engineers, SPE International Conference and Exhibition on Health, Safety, Security, Environment and Social Responsibility, Abu Dhabi, UAE, 16-18 April 2018

[4] S. Doz, P.Y. Foucher, ONERA; X. Watremez, TOTAL, "Methane leak near real time quantification with a hyperspectral infrared camera", In Proceedings of SPIE Defense and Commercial Sensing, Orlando, USA, 15-18 April 2018

[5] N. Cézard, S. Le Méhauté, P. Benoit, D. Fleury, D. Goular, J. Le Gouet, C. Planchat, A. Dolfi-Bouteyre, ONERA ; X. Watremez, TOTAL, "All-fiber DIAL/DOPPLER Lidar at 1.65 micron for remote detection of methane leaks", In Proceedings of International Laser Radar Conference, 29th, Hefei, China, 24-28 June 2019

[6] J.B. Dherbecourt, J. M. Melkonian, A. Godard, V. Lebat, N. Tanguy, C. Blanchard, M. Raybaut, ONERA; X. Watremez, D. Dubucq, TOTAL, "The NAOMI GAZL multispecies differential absorption lidar: realization and testing on the TADI gas leak simulation facility", Conference on Lasers and Electro-Optics, San José, USA, 5-10 May 2019 